\title{
Clinical subtyping and early detection: short-term challenges for child and adolescent psychiatry
}

\author{
Celso Arango
}

(C) Springer-Verlag 2010

Very recently in this same journal, Michael Rutter reviewed the most important future opportunities and challenges for child and adolescent psychiatry [1]. Those opportunities clearly include early detection and clinical subtypes to reduce heterogeneity at the syndrome level. Two of the articles in this issue deal with these important topics.

As in many other mental disorders, early diagnosis and treatment of autism spectrum disorders (ASD) improve prognosis. Early detection can provide access to specific therapeutic approaches within a critical therapeutic window not only in severe cases but also in less severely affected children. Recently, efforts have focused on early diagnosis in children who are younger than 2 years of age. Previous reports show that health surveillance programmes can identify infants at risk for mental health problems in the first few months of life if standardised measures and specific training of the health-care professionals are provided [2].

Recently, the Centers for Disease Control and Prevention (CDC) estimated that an average of 1 in 110 children in the US have an ASD [3]. What not long ago used to be a rare disorder is now a spectrum with prevalence close to the more common mental disorders in childhood. This increase in prevalence makes ASDs an urgent public health concern. Now more than ever, research is needed to ascertain who is at risk and what factors put certain persons

\section{Arango ( $\square)$}

Unidad de Adolescentes, Departamento de Psiquiatría, Hospital General Universitario Gregorio Marañón, Centro de Investigación Biomédica en Red de Salud Mental CIBERSAM, C/Ibiza, 43, 28009 Madrid, Spain

e-mail: carango@hggm.es at risk in order to improve long-term outcome in these patients and their families.

Möricke et al. [4] assessed the relationship between early subclinical autistic traits (in three groups with high, moderate, and low scores for autistic traits) and the occurrence of later developmental and behavioural problems, as well as language and cognitive impairments. Although the primary outcome of this study seemed to be the prediction of autistic symptoms and/or ASD diagnoses at age 4-5 years through detection of autistic traits at age 14-15 months, none of the children developed an ASD disorder and those with high scores for autistic traits did not show continuity at the 4- to 5-year follow-up. This may be well due to the small sample size of the study. More interesting is the finding that children in the high autistic traits group showed more externalising and internalising behavioural problems as well as language and cognitive impairments than children in the other groups at age 3 years. The main implications of this finding are that early social and communication skills predict symptoms in the sphere of self-regulation of emotions and impulses. On a more practical level, the study shows how early recognition of children at risk for developing behavioural, language, and cognitive problems is possible.

Heterogeneity is certainly one of the major obstacles in child and adolescent psychiatry. Although this also holds true for adult psychiatry, the rate of comorbidity seems to be higher in the paediatric population than in adults. The search for clinical phenotypes to reduce heterogeneity in diseases such as OCD has been the aim of many research groups in recent years. This approach has been used in other disorders and behaviours such as suicide [5] and at the symptomatic level as is the case of insight [6]. Langley et al. [7] report on a clinically relevant study 
with a very large group of OCD patients. The approach taken by these authors is to assess different OCD groups based on their comorbidities with externalising or anxiety disorders.

The identification of distinct subgroups, in this case based on comorbidity, is an interesting approach to studying variations in age of OCD onset, symptom expression and course, family/genetics, and treatment response. Although the paper published in this issue has some limitations, such as the wide range of patient ages (5-17), the cross-sectional nature of the study, and the lack of inclusion of patients with OCD and other comorbidities, the study has relevant clinical results. Greater OCD severity was present in patients with co-occurring anxiety disorder, and children with comorbid externalising disorders showed lower overall functioning. The identification of such subgroups may be relevant for future studies assessing specific therapeutic interventions, such as cognitive therapy, that may be more efficient for some clinically identified subgroups than others [8]. When all patients with a heterogeneous disorder such as OCD are included in a clinical trial, we may reduce the likelihood of detecting therapeutic interventions that are efficacious in certain subgroups of patients. As a speculative example only, it could be the case that a behavioural approach may be more efficacious for patients with externalising comorbidity and a cognitive approach for patients with a more pure OCD.

\section{References}

1. Rutter M (2010) Child and adolescent psychiatry: past scientific achievements and challenges for the future. Eur Child Adolesc Psychiatry. doi:10.1007/s00787-010-0111-y

2. Skovgaard AM, Houmann T, Christiansen E, Olsen EM, Landorph SL, Lichtenberg A, Jorgensen T (2008) Can a general health surveillance between birth, 10 months identify children with mental disorder at 1(1/2) year? A case-control study nested in cohort CCC 2000. Eur Child Adolesc Psychiatry 17(5):290-298

3. Prevalence of autism spectrum disorders-autism and developmental disabilities monitoring network, United States, 2006 (2009). MMWR Surveill Summ 58(10):1-20

4. Möricke E, Swinkels SH, Beuker KT, Buitelaar JK (2010) Predictive value of subclinical autistic traits at age 14-15 months for behavioural and cognitive problems at age 3-5 years. Eur Child Adolesc Psychiatry. doi:10.1007/s00787-010-0103-y

5. Hawton K, Harriss L, Rodham K (2010) How adolescents who cut themselves differ from those who take overdoses. Eur Child Adolesc Psychiatry 19(6):513-523

6. Parellada M, Fraguas D, Bombin I, Otero S, Castro-Fornieles J, Baeza I, Gonzalez-Pinto A, Graell M, Soutullo C, Paya B, Arango C (2009) Insight correlates in child- and adolescent-onset first episodes of psychosis: Results from the CAFEPS study. Psychol Med 39(9):1433-1445

7. Langley AK, Lewin AB, Bergman RL, Lee JC, Piacentini J (2010) Correlates of comorbid anxiety and externalizing disorders in childhood obsessive compulsive disorder. Eur Child Adolesc Psychiatry. doi:10.1007/s00787-010-0101-0

8. Williams TI, Salkovskis PM, Forrester L, Turner S, White H, Allsopp MA (2010) A randomised controlled trial of cognitive behavioural treatment for obsessive compulsive disorder in children and adolescents. Eur Child Adolesc Psychiatry 19(5):449-456 